\title{
Introgression the Salinity Tolerance QTLs Saltol into AS996, the Elite Rice Variety of Vietnam
}

\author{
Luu T. N. Huyen ${ }^{1 *}$, Luu M. Cuc ${ }^{1}$, Abdelbagi M. Ismail ${ }^{2}$, Le H. Ham ${ }^{1}$ \\ ${ }^{1}$ Agricultural Genetics Institute; Vietnam Academy of Agricultural Sciences, Hanoi, Vietnam; ${ }^{2}$ International Rice Research Institute, \\ Metro Manila, Philippine. \\ Email: "huyenluu116@gmail.com,LHHAM@agi.ac.vn
}

Received February 20 ${ }^{\text {th }}, 2012$; revised March $8^{\text {th }}, 2012$; accepted April $19^{\text {th }}, 2012$

\begin{abstract}
This study focus on developing new salinity tolerance and high yielding rice lines, using markers assisted backrossing (MABC). Total of 500 SSR markers on 12 rice chromosomes were screened for parental polymorphic markers. Of which, 52 primers in the Saltol region were checked with the two parents varieties to identify polymorphic primers for screening the Saltol region of the breeding populations. For each backcross generation of ASS996/FL478, approx. 500 plants were screened with 63 polymorphic markers distributed on 12 chromosomes. The two $\mathrm{BC}_{1} \mathrm{~F}_{1}$ plants $\mathrm{P} 284$ and P307 which had the highest recipient alleles up to $89.06 \%$ and $86.36 \%$, were chosen for the next backcrossing. Three $\mathrm{BC}_{2} \mathrm{~F}_{1}$ plants with the recipient alleles up to $94.03 \%$ and $93.18 \%$ were used to develop $\mathrm{BC}_{3} \mathrm{~F}_{1}$ generation. The best BC3F1 plant was P284-112-209 with all the recipient alleles and Saltol region. The four plants P307-305-21, P284112-195, P284-112-198, P284-112-213 were the second ranking with only one loci heterozygous (applied 63 markers covered on 12 chromosomes). These five plants were chosen as the breeding lines for result of Saltol-AS996 introgression. The breeding line $\mathrm{BC}_{4} \mathrm{~F}_{1}$ having $100 \%$ genetic background of donor variety is ready for develop new salinity tolerant variety ASS996-Saltol to cope with climate change.
\end{abstract}

Keywords: Marker Assisted Backcrossing; Rice Variety AS996; Salinity Tolerance-Saltol; QTLs

\section{Introduction}

Rice is the most important food source for half of the world's population and also the main staple food for most of the country's 86 millions people. Vietnam is the world's second-largest rice exporter, along with the top exporter Thailand, counted for 50 percent of the world rice trade. Developing adaptation rice varieties to cope with climate change and sea level rise for the Red River Delta and Mekong River Delta is crucial to Vietnam economy and food security, it also contributes to the global food security.

Research at IRRI resulted in the development of high yielding rice varieties tolerant of abiotic stresses such as submergence and salt stress, and these varieties can help the unfavorable coastal areas less vulnerable to climate change impacts [1]. These improved varieties were developed using both conventional and modern breeding tools. Breakthroughs in salinity tolerance breeding became feasible after the identification of major chromosomal regions (Quantitative trait loci, QTLs) underlining salinity (Saltol) stresses, the development and use of a marker system for their speedy incorporation into mod-

\footnotetext{
"Corresponding author.
}

ern high yielding and popular varieties through marker assisted backcrossing (MABC) [1]. The basis of MABC strategy is to transfer a gene/QTL from a donor line to a recipient line while selecting against donor introgressions across the rest of the genome [1-3]. MABC is a precise and effective method to introgress a single locus controlling a trait of interest while retaining the essential characteristics of the recurrent parent [4]. MABC has three main advantages over conventional backcrossing. Firstly, DNA markers can be used for simple and efficient selection of the target locus ("foreground selection"). Secondly, the size of the donor chromosome segment containing the target locus can be minimized ("recombinant selection"). Thirdly, the recovery of the recurrent parent can be accelerated by selecting backcross lines with a higher proportion of recurrent parent genome ("background selection").

In this study, MABC breeding strategy was applied for develop new salt tolerance Vietnam's varieties. We have already applied MABC including foreground selection, recombinant selection folowed by background selection in all the backcross generation $\mathrm{BC}_{1} \mathrm{~F}_{1}, \mathrm{BC}_{2} \mathrm{~F}_{1}$ and $\mathrm{BC}_{3} \mathrm{~F}_{1}$ of the cross AS996/FL478.

The recipient rice variety AS996 was widely grown in 
South of Vietnam. AS996 variety was derived from the cross IR64/Oryzarufipugon, official released in 2002. The yield potential of this variety is 8 ton/ha under optimum management.

The donor rice variety FL478 (IR 66946-3R-178-1-1), has been promoted as an improved donor for breeding programs, as it has a high level of seedling stage salinity tolerance and is photoperiod insensitive, shorter and flowers earlier than the original Pokkali landrace. Saltol is a major QTL associated with the Na-K ratio and seedling-stage salinity tolerance, was identified on chromosome 1. This QTL was tested in a hydroponic screen at the seedling stage revealed that this QTL explained 43\% of the variation for seedling shoot $\mathrm{Na}-\mathrm{K}$ ratio and salinity tolerance in the population [4]. Furthermore, an analysis of single feature polymorphism in the Saltol region suggested that FL478 contained a DNA fragment smaller than $1 \mathrm{Mb}$ from Pokkali at $10.6-11.5 \mathrm{Mb}$ on chromosome $1[5]$.

\section{Materials and Methods}

1) Plant materials and crossing scheme

FL478 was used as the donor of Saltol. The recipient varieties were AS996, which is widely grown cultivars in the South of Vietnam.

For the MABC breeding strategy, AS996 was crossed with FL478 to obtain F1 seeds. F1 was backcrossed to AS996 to obtained a large number of BC1F1. 573 $\mathrm{BC} 1 \mathrm{~F} 1$ plants were screened for foreground, recombinant and background selections. The plants carrying target QTL and the biggest recepient genom were selected for the next $\mathrm{BC}$ generation. Over five hundreds $\mathrm{BC} 2 \mathrm{~F} 1$ and then $371 \mathrm{BC} 3 \mathrm{~F} 1$ plants were screened for foreground, recombinant and background selections. The $\mathrm{BC} 2 \mathrm{~F} 2$ or $\mathrm{BC} 3 \mathrm{~F} 1$ individuals carrying target genes and almost recepient genom were obtained.

2) Parents SSR polymorphism screening: Approx. 500 SSR markers distributed in the 12 chromosomes including foreground, recombinant and background markers were screened.

3) Genotype data was obtained by analysing DNA with SSR markers using $15 \mu \mathrm{L}$ PCR reactions on 96-well plates. After initial denaturation for $4 \mathrm{~min}$ at $94^{\circ} \mathrm{C}$ each cycle comprised $1 \mathrm{~min}$ denaturation at $94^{\circ} \mathrm{C}, 1 \mathrm{~min}$ annealing at $55^{\circ} \mathrm{C}$, and $1 \mathrm{~min}$ extension at $72^{\circ} \mathrm{C}$ with a final extension for $5 \mathrm{~min}$ at $72^{\circ} \mathrm{C}$ at the end of 30 cycles (Eppendoft thermal cyclers). The PCR products were mixed with bromophenol blue gel loading dye and were analyzed by electrophoresis on $4.5 \%$ acrylamide gel at 1500 $\mathrm{V}$ (Biorad system) followed by silver stainning steps and scoring; or electrophoresis on $6 \%-8 \%$ acrylamide gels at $100 \mathrm{v}$ (Dual Triple-Wide Mini-Vertical System, C. B. S. Scientific, CA, USA) followed by SYBR-Safe staining
(Invitrogen), gel documentation (Alpha Innotech), and manual scoring of the gel pictures).

4) Data analysis

The molecular weights of the different alleles were measured using Alpha Ease Fc 5.0 software. The marker data was analyzed using the software Graphical Genotyper (GGT 2.0) [6]. The homozygous recipient allele, homozygous dominant allele and heterozygous allele were scored as "A", "B" and " $\mathrm{H}$ ". The percent markers homozygous for recipient parent $(\% \mathrm{~A})$ and the percent recipient alleles including heterozygous plants (\%R) were calculated.

5) Evaluation of salinity tolerance

Pre-germinated $\mathrm{BC} 2 \mathrm{~F} 2$ and $\mathrm{BC} 3 \mathrm{~F} 1$ seeds were sown in holes on styrofoam floats with a net bottom suspended on trays filled with Yoshida nutrient solution [7]. Three replications were used for each experiment, with nine individual plants per line evaluated for each replication. Salt stress was imposed 14 days after germination by adding $\mathrm{NaCl}$ to an $\mathrm{EC}$ of $12 \mathrm{dS} \cdot \mathrm{m}^{-1}$ in Yoshida nutrient solution until final scoring. IR29 (sensitive) and FL478 (highly tolerant) were used as checks. The $\mathrm{pH}$ of the nutrient solution was adjusted daily to 5.0, and the culture solutions were replaced every 5 days. Entries were scored based on visual symptoms using IRRI's Standard Evaluating Score (SES) for rice, with ratings from 1 (highly tolerant) to 9 (highly sensitive) [8].

\section{Results and Discussion}

\subsection{Parental SSR Polymorphism Screening}

A number of about 500 SSR markers on 12 rice chromosomes were screened for parental polymorphic markers for all foreground, recombinant and background analyses. On the Figure 1, we screened 8 rice varieties (Q5, Q5DB, OM5472, FL478, IR64Sub1, AS996, KDDB, BT) to found out the polymorphic markers to use in genotyping the backcross populations.

Total of 63 polymorphic markers for the cross AS996/ FL478 were identified. The frequence of polymorphic SSR markers between the two parents AS996/FL478 was $12.6 \%$ only. The list of polymorphic markers was described in Table 1.

All those markers were used for screening the $\mathrm{BC} 1 \mathrm{~F} 1$, $\mathrm{BC} 2 \mathrm{~F} 1$ and $\mathrm{BC} 3 \mathrm{~F} 1$ generations.

\subsection{Genotyping}

\subsubsection{Genotyping BC1F1}

Based on the map of Saltol QTL region, the best markers within the Saltol QTL region were AP3206 and RM3412, the most useful markers flanking the Saltol region were RM10694 (telomeric to Saltol) and RM493 and RM 10793 (centromeric to Saltol), while nearby markers that 


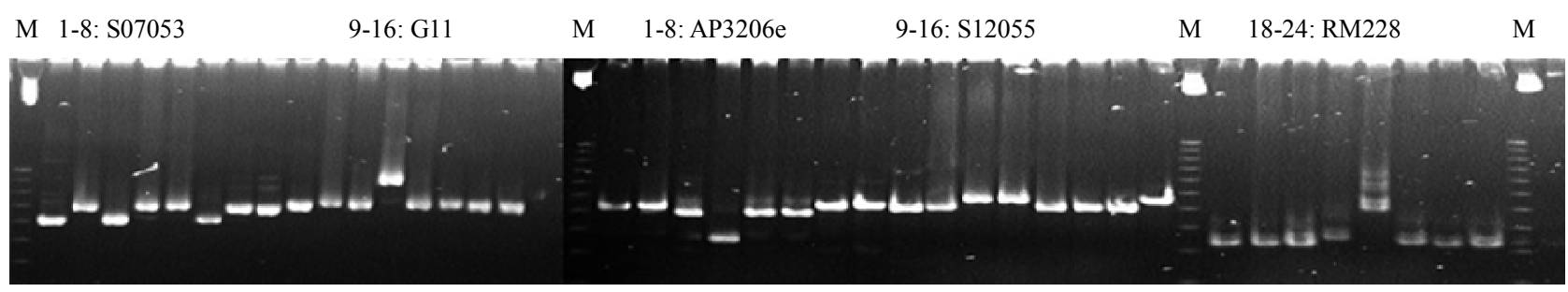

Figure 1. Parental screening on 6\% polyacrylamide gel. DNA for each primer: 1. Q5; 2. Q5DB; 3. OM5472; 4 . FL478; 5. IR64SUB1; 6. AS996; 7. KDDB; 8. BT; M: 25 bp ladder. Primers: SO7053; G11; AP3206e; S12055; RM228.

Table 1. Polymorphic markers were used.

\begin{tabular}{|c|c|c|c|c|c|c|c|}
\hline No & Primers & Chro. & $\mathrm{Mb}$ & Forward sequences & Reverse sequences & Size & Tann. \\
\hline 1 & RM10287 & 1 & 5 & GTATTCCTTGCTGCTGCTGATGG & GACTGGAGATGTGATCGGAAACC & 184 & 60 \\
\hline 2 & G11A & 1 & 9.3 & AGCTGGTAGGAAGGCTGAAAG & TGCCAGCAGCTCAGTAGAAG & 250 & 57 \\
\hline 3 & RM10694 & 1 & 11 & TTTCCCTGGTTTCAAGCTTACG & AGTACGGTACCTTGATGGTAGAAAGG & 195 & 60 \\
\hline 4 & AP3206 & 1 & 11.2 & GGAGGAGGAGAGGAAGAAG & gcaagaattaatccatgtgaaaga & 180 & 60 \\
\hline 5 & RM3412 & 1 & 11.5 & TGATGGATCTCTGAGGTGTAAAGAGC & TGCACTAATCTTTCTGCCACAGC & 215 & 55 \\
\hline 6 & RM10711 & 1 & 11.2 & GCTTCGATCGATGAGAAAGTAGAGG & GAATCTCССАТССТTСССТTCC & 173 & 60 \\
\hline 7 & RM493 & 1 & 12.3 & GTACGTAAACGCGGAAGGTGACG & CGACGTACGAGATGCCGATCC & 178 & 55 \\
\hline 8 & RM10793 & 1 & 12.5 & GACTTGCCAACTCCTTCAATTCG & TCGTCGAGTAGCTTCCCTCTCTACC & 124 & 60 \\
\hline 9 & RM562 & 1 & 14.6 & GGAAAGGAAGAATCAGACACAGAGC & GTACCGTTCCTTTCGTCACTTCC & 126 & 55 \\
\hline 10 & RM7075 & 1 & 15.1 & GCGTTGCAGCGGAATTTGTAGG & CCCTGCTTCTCTCGTGCAGTCG & 376 & 55 \\
\hline 11 & RM11125 & 1 & 20.5 & CCAAGAACCCTAGCTCССТCTCC & TCGACGAGATCCTCCTCGTAAACC & 212 & 60 \\
\hline 12 & RM7643 & 1 & 31.1 & AAACCGCCGTCCTCCTATTCG & CTTGAGCGCACCAACGAAATACC & 178 & 55 \\
\hline 13 & SO1132A & 1 & 32 & CAATGACGACGCATGTATGT & TGCTTGAATGTTTTTCGAGGA & 180 & 55 \\
\hline 14 & RM3482 & 1 & 39.7 & GCCGCTAATGTTGTTGTCAAGC & CGAAGCCAACGTAGTCCAATCC & 173 & 55 \\
\hline 15 & RM300 & 2 & 13.2 & GGGCTTAAGGACTTCTGCGAACC & AGCGATCCACATCATCAAATCG & 162 & 55 \\
\hline 16 & RM13197 & 2 & 16.5 & AAACCCTCCGGCTCATTCTTGC & ACTCGAATCGTATCGGCTTGAGG & 183 & 60 \\
\hline 17 & RM341 & 2 & 19.2 & CAAGAAACCTCAATCCGAGC & CTCCTCCCGATCCCAATC & 200 & 55 \\
\hline 18 & RM6318 & 2 & 24.4 & AAGTGCCTCGAATTACACATCTCC & GCTGCTTCTGTCCAGTGAGACC & 188 & 55 \\
\hline 19 & RM13628 & 2 & 25.1 & TATGCCACGAATGACCCTAACC & CTCCATATGCAGCGACAATCG & 200 & 60 \\
\hline 20 & RM231 & 3 & 2.5 & CCAGATTATTTCCTGAGGTC & CACTTGCATAGTTCTGCATTG & 170 & 55 \\
\hline 21 & RM3297 & 3 & 13.4 & TGCACGTGATCTCTTGTAACCTAGC & GGAGAGGGCCTTGTTCTTGAGG & 298 & 55 \\
\hline 22 & SO3065 & 3 & 15 & TTTCGTGCGGGGATATAGAG & GCAAGATAACTCAAAATCAAAAGC & 180 & 55 \\
\hline 23 & SO3068 & 3 & 17 & TTGACAAGTTTTGGAAATTGGA & TTGTTGTGCCATTGGAGAAA & 195 & 55 \\
\hline 24 & SO3072 & 3 & 19 & TTTCGTGCGGGGATATAGAG & GCAAGATAACTCAAAATCAAAAGC & 167 & 55 \\
\hline 25 & RM7097 & 3 & 26.7 & GGCCATTATGTGCATCTCTCAGC & GGATCGATCGACATCAATCTTGG & 177 & 55 \\
\hline 26 & RM518 & 4 & 2 & AAGACACAAGCAAACAGCTCAACC & AAGCTTGCTTGGTTCAAGAGAGG & 193 & 55 \\
\hline 27 & RM5639 & 4 & 8.1 & AGGAGGAAGGAAGAACAGAGTTGC & CTGAGTGCGTGCCATTTATTTCC & 139 & 55 \\
\hline
\end{tabular}




\section{Continued}

\begin{tabular}{|c|c|c|c|c|c|c|c|}
\hline 28 & R4M17 & 4 & 11.5 & AGTGCTCGGTTTTGTTTTC & GTCAGATATAATTGATGGATGTA & 220 & 55 \\
\hline 29 & RM307 & 4 & 12.9 & gtactaccgacctaccgttcac & ctgctatgcatgaactgctc & 290 & 55 \\
\hline 30 & RM5626 & 4 & 24.7 & GGACGCCACCTTCCTCTTCTGC & CGGTCATAAACGCCATTAGACCAAGC & 97 & 55 \\
\hline 31 & RM6329 & 4 & 28.7 & CAGCAGAGACTATAGACACTCAAGC & TGCCTAGCTACTCTAGGTGAAACC & 344 & 55 \\
\hline 32 & RM3867 & 4 & 31.5 & TCCTCCTCACTCGATCATAATGC & TTCTCCTACTTGACTGGAACATCG & 187 & 55 \\
\hline 33 & RM127 & 4 & 34.8 & CGAAGCTTTCGGTGGGATAGC & ACCTTGAGCGAGTCCTTGAACG & 195 & 55 \\
\hline 34 & RM437 & 5 & 3.8 & АТСССТССТСТGСTCAATGTTGG & TCAGGGAGGGTCCTAGCTACTGG & 183 & 55 \\
\hline 35 & RM122 & 5 & 6.3 & GAGTCGATGTAATGTCATCAGTGC & GAAGGAGGTATCGCTTTGTTGGAC & 200 & 55 \\
\hline 36 & RM249 & 5 & 10.7 & ggcgtaaaggttttgcatgt & atgatgccatgaaggtcagc & 135 & 55 \\
\hline 37 & RM163 & 5 & 18.9 & CGCCTTTATGAGGAGGAGATGG & AAACTCTTCGACACGCCTTGC & 179 & 55 \\
\hline 38 & RM18877 & 5 & 23.5 & ACCACTGCTGCAAAGAACATTGG & GCGAGAATAAGATGAGACACAAGAGG & 191 & 60 \\
\hline 39 & RM510 & 6 & 2.8 & GTTTGACGCGATAAACCGACAGC & ATGAGGACGACGAGCAGATTCC & 193 & 55 \\
\hline 40 & RM585 & 6 & 3.2 & CTAGCTAGCCATGCTCTCGTACC & CTGTGACTGACTTGGTCATAGGG & 174 & 55 \\
\hline 41 & SO6065A & 6 & 16.5 & cccettcatcattgcaactt & agtctctccatcacccgtct & 190 & 55 \\
\hline 42 & RM3635 & 7 & 11.1 & GAGAGACAGTGGAAGGGAAGACG & GTTCССТСССТССТCСТAGTTCG & 90 & 55 \\
\hline 43 & SO 7053 & 7 & 15.3 & cgaaactttgggacgaaatg & cgtccaccattcactgtcac & 210 & 55 \\
\hline 44 & RM455 & 7 & 22.3 & CCACAAATTAATCCGGATCACACC & AGCATTGTGCAATCACGAGAAGG & 175 & 55 \\
\hline 45 & RM337 & 8 & 0.1 & GTAGGAAAGGAAGGGCAGAG & CGATAGATAGCTAGATGTGGCC & 200 & 55 \\
\hline 46 & RM152 & 8 & 0.6 & AAGGAGAAGTTCTTCGCCCAGTGC & GCCCATTAGTGACTGCTCCTAGTCG & 160 & 55 \\
\hline 47 & RM310 & 8 & 5 & GACTTGTGGTTGTTGCTTGTTGG & ACTGCCATATGCATTTCCCTAGC & 176 & 55 \\
\hline 48 & RM547 & 8 & 5.5 & TTGTCAAGATCATCCTCGTAGC & GTCATTCTGCAACCTGAGATCC & 283 & 55 \\
\hline 49 & RM23877 & 9 & 6.5 & TGCCACATGTTGAGAGTGATGC & TACGCAAGCCATGACAATTCG & 328 & 60 \\
\hline 50 & R9M30 & 9 & 14.6 & СТСАССТАССТААААСССААС & CCACCCAAATCTGATACTG & 170 & 55 \\
\hline 51 & RM215 & 9 & 20.9 & GAGCAGCAAGAGCAGCAGAGG & CATGCTCGACTTCAGAAGCTTGG & 174 & 55 \\
\hline 52 & RM171 & 10 & 2.6 & AACGCGAGGACACGTACTTAC & ACGAGATACGTACGCCTTTG & 360 & 55 \\
\hline 53 & RM 25022 & 10 & 3.6 & ACATTCCGCGTTTGTGTGTAGC & GCTTGGTAGTTGGGCTGATGG & 200 & 60 \\
\hline 54 & R10M10 & 10 & 4.9 & GAATACAACCCCCTAAAAAC & ATGGACCGTTGAGGAGAC & 200 & 55 \\
\hline 55 & RM25271 & 10 & 10.7 & AGACGCTACTCCCACCTGTAACC & ATATCATTGCCGCAACACAAGC & 186 & 60 \\
\hline 56 & RM21 & 11 & 19.1 & ACAGTATTCCGTAGGCACGG & GCTCCATGAGGGTGGTAGAG & 160 & 55 \\
\hline 57 & RM206 & 11 & 21.8 & ATCGATCCGTATGGGTTCTAGC & GTCCATGTAGCCAATCTTATGTGG & 200 & 55 \\
\hline 58 & RM224 & 11 & 27.1 & ATCGATCGATCTTCACGAGG & TGCTATAAAAGGCATTCGGG & 220 & 55 \\
\hline 59 & $\mathrm{~S} 11117 \mathrm{C}$ & 11 & 29.5 & caaccatgtctatgatcgatgt & ggctgtctccatgttgaggt & 180 & 60 \\
\hline 60 & RM7102 & 12 & 13.3 & GGGCGTTCGGTTTACTTGGTTACTCG & GGCGGCATAGGAGTGTTTAGAGTGC & 212 & 55 \\
\hline 61 & S12055 & 12 & 15.2 & aaacggattagaggggatcg & aagaaagttccttcatgaggctat & 160 & 55 \\
\hline 62 & RM28746 & 12 & 26.4 & GAAGAAAGAAGACGCCAAGAAACG & САТТССАТТСССТТССТСТTCG & 160 & 55 \\
\hline 63 & RM17 & 12 & 26.9 & TGCCCTGTTATTTTCTTCTCTC & GGTGATCCTTTCCCATTTCA & 300 & 55 \\
\hline
\end{tabular}


can be used for negative selection are RM490 above Saltol and RM7075 below. Microsatellite markers unlinked to Saltol covering all the chromosomes, that were polymorphic between the two parents, were used for recombinant and background selection to recover the recipient genome. Among 63 parents polymorphic markers, 42 markers were analysed for selection initially on $\mathrm{BC} 1 \mathrm{~F} 1$ individuals. For foreground selection, AP3206, RM3412 and RM10793 were used for screening heterozygous plants (Figure 2). After that step, another flanking markers were used to identified the recombinant plants.

Total of 12 recombinant plants were used for background selection. Two plants P284 and P307 had the highest recipient alleles up to $89.06 \%$ and $86.36 \%$ were used to develop BC2F1 populations (Table 2). In case the ordinary breeding was applied, the frequency of recipient genome was only $75 \%$ in the $\mathrm{BC} 1 \mathrm{~F} 1$, lower than in this study $16 \%-19 \%$.

\subsubsection{Genotyping BC2F1}

Approx. five hundred $\mathrm{BC} 2 \mathrm{~F} 1$ individuals of the cross (AS996xFL478) was grown and analysed. The same procedures were applied to screen the foreground selection again with AP3206, RM3412, RM10793, RM10711 (Figure 3). The recombinant selection was done with RM10694, RM562, RM7075 along the Saltol region on chromosome 1. From 250 heterozygous plants, 26 recombinant plants were identified.

For background selection, the primers shown heterozygou DNA bands from previous generation with 10 more primers additional were used. Plant P307-322, P284-112 and P307-305 were the best plants with the recipient alleles up to $93.18 \%$ and $94.03 \%$ respectively. These three plants were used to cross with recipient variety for BC3F1 generation. In each individuals, half of the tillers were used for $\mathrm{BC} 3 \mathrm{~F} 1$ crossing, the others were used for $\mathrm{BC} 2 \mathrm{~F} 2$ selfing. In case the ordinary breeding were applied, frequency of receipient genome was only $87.5 \%$ in the $\mathrm{BC} 1 \mathrm{~F} 1$, but in here, the best plants were selected with the recipient alleles about 5.7\% - 6.5\% higher than conventional method.

\subsubsection{Genotyping BC3F1}

From the above results, three populations from three plants were analysed. Total of 371 plants were screened for the four markers located in the Saltol region. Only 94 plants were used for recombinant selection. In background selection, 25 primers were used. Most of used primers amplified the DNA fragments on other position in remain chromosomes (except for the QTLs fragment on chromosome 1) having the band of recurrent parent. They were convinced in Figure 4: Using primer RM510, RM585, RM3297 to screen 14 BC3F1 plants, the results were the last lanes in Figures 4(a)-(c) were the band of FL478, while all of $\mathrm{BC} 3 \mathrm{~F} 1$ plants having the same band of recurrent parent AS996.

Plant P284-112-209 was the best BC3F1 individual with all the recipient alleles screened based on total of 63 markers (Figures 5, 6). The four plants P307-305-21, P284-112-195, P284-112-198, P284-112-213 were the second ranking with only one loci heterozygous. All those 5 plants were chosen as the breeding lines for result of Saltol-AS996 introgression.

\subsubsection{Evaluation of Salinity Tolerance}

As described in the part of method, $\mathrm{BC} 2 \mathrm{~F} 2$ and $\mathrm{BC} 3 \mathrm{~F} 1$ seeds were screening to evaluate the introgression of Saltol fragment into AS996. Salt stress was imposed 14 days after germination by adding $\mathrm{NaCl}$ to an $\mathrm{EC}$ of 12 $\mathrm{dS} \cdot \mathrm{m}^{-1}$ in Yoshida nutrient solution until final scoring.

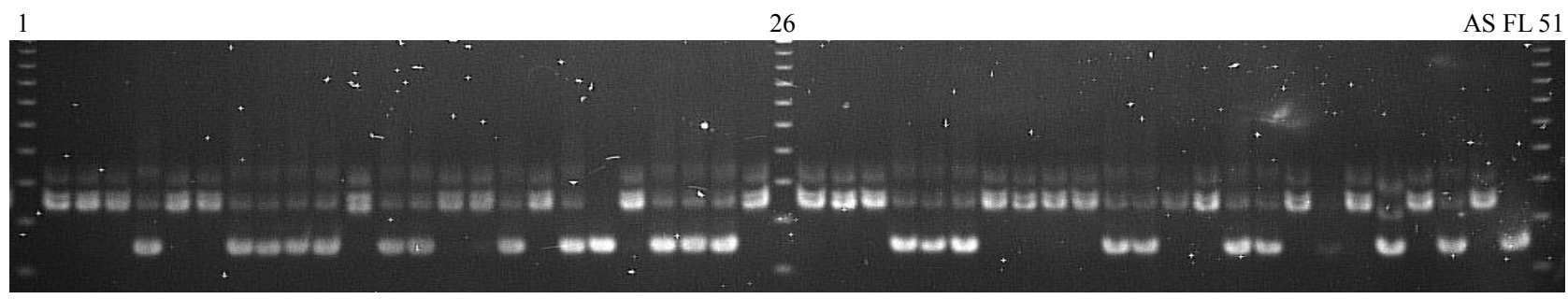

Figure 2. Screening individuals on crossed BC1F1 (AS996/FL478) using primer RM310. Wells 1, 26, 51: 25 bp marker, 2 - 25 and 27 - 48: BC1F1 in dividuals, Well 49: AS996, Well 50: FL478.

Table 2. The recippient allele of the twelves BC1F1 recombinant plants.

\begin{tabular}{ccccccccccccc}
\hline Plant number & 65 & 149 & 228 & 238 & 281 & $\mathbf{2 8 4}$ & 305 & $\mathbf{3 0 7}$ & 311 & 401 & $\mathbf{4 1 1}$ & 426 \\
\hline A & 55.26 & 51.43 & 60.53 & 44.74 & 56.25 & $\mathbf{7 8 . 1 3}$ & 66.67 & $\mathbf{7 5 . 7 6}$ & 63.64 & 73.68 & $\mathbf{6 6 . 6 7}$ & 63.64 \\
H & 34.38 & 37.93 & 36.36 & 34.38 & 15.63 & $\mathbf{2 1 . 8 8}$ & 33.33 & $\mathbf{2 1 . 2 1}$ & 36.36 & 0.00 & $\mathbf{3 3 . 3 3}$ & 36.36 \\
R & 72.45 & 70.39 & 78.71 & 61.92 & 64.06 & $\mathbf{8 9 . 0 6}$ & 83.33 & $\mathbf{8 6 . 3 6}$ & 81.82 & 73.68 & $\mathbf{8 3 . 3 3}$ & 81.82 \\
\hline
\end{tabular}




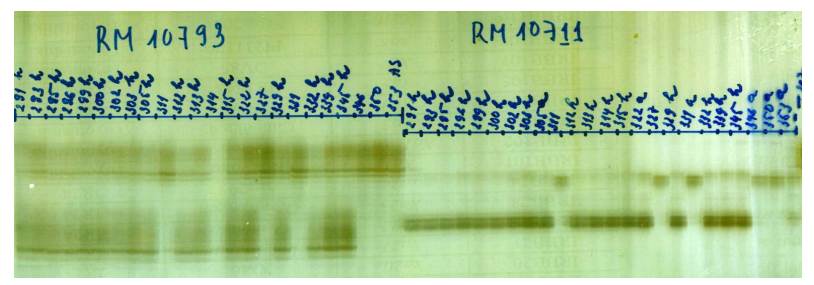

Figure 3. BC2F1 (AS996xFL478) individuals screening using primer RM10793-left, and RM10711-right.

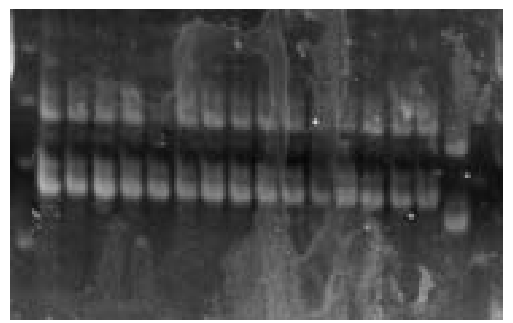

(a)

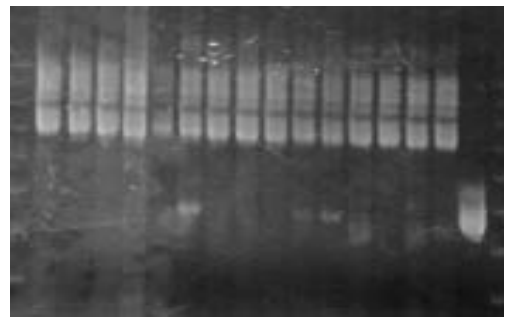

(b)

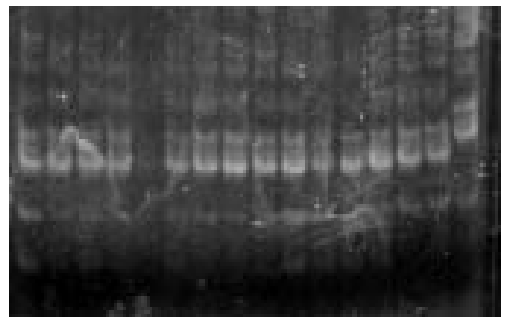

(c)

Figure 4. Background selection with 14 recombinant BC3F1 plants using primers RM510, RM585, RM3297.

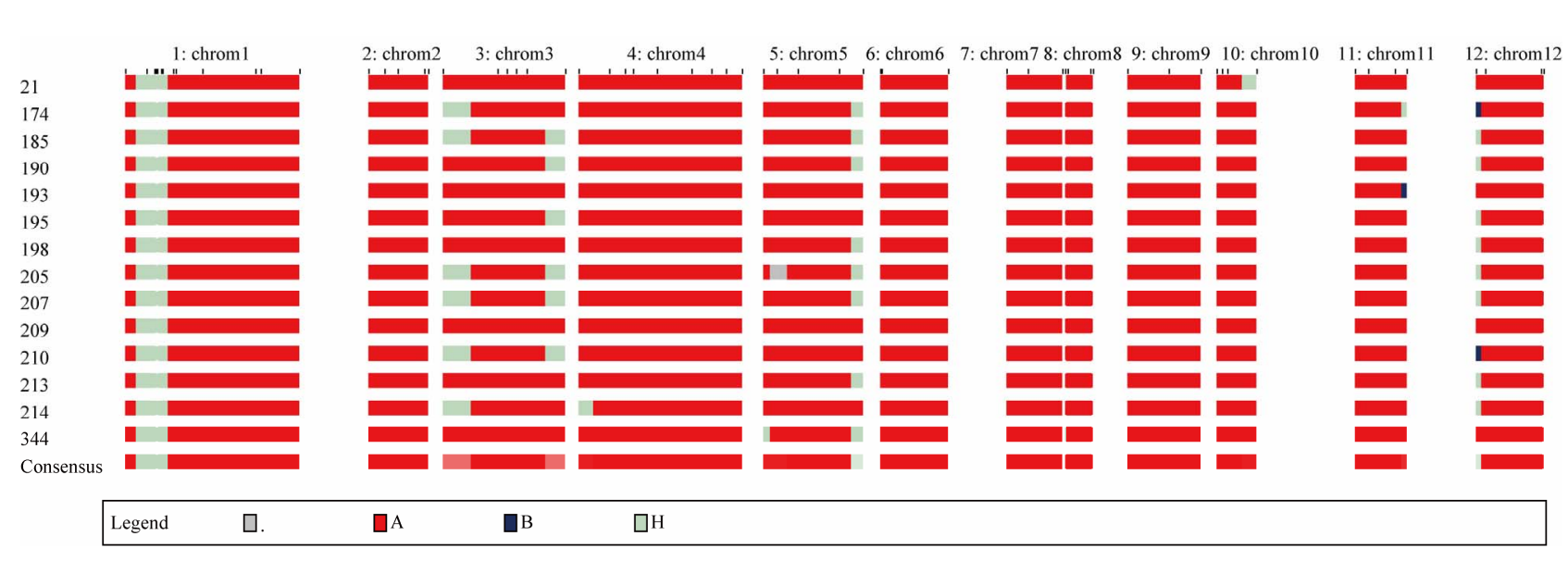

Figure 5. Graphical of the 14 recombinant plants BC3F1 using GGT2.0.
Based on visual symptoms using IRRI's SES for rice, when the suceptible variety IR29 (sensitive) scored 9 and variety FL478 were used as highly tolerant checks scored 3 , all the BC2F2 of the selected plants P284-112, P307305 and P307-322 having the same score as the tolerant checks. It means that the homozygous Saltol fragment working well in $\mathrm{BC} 2 \mathrm{~F} 2$ generation. The $\mathrm{BC} 3 \mathrm{~F} 1$ plants scored 3 - 5 because of the heterozygous of Saltol fragment. The result of Salinity tolerance screening shown that, all the BC2F2 of the selected plants P284-112, P307-305 and P307-322 having the same score as the tolerant check. It means the homozygous Saltol fragment working well in BC2F2 generation. The next generation will be used to check the function of Saltol in the following $\mathrm{BC} 3 \mathrm{~F} 2, \mathrm{BC} 2 \mathrm{~F} 3$.

\section{Conclusions}

- Approx 500 SSR markers distributed in the 12 chromosomes were screened for parental polymorphism. Of which, 63 polymorphic markers were identified.

- Two BC1F1 plants P284 and P307, had the highest recipient alleles up to $89.06 \%$ and $86.36 \%$, were identified for the next backcrossing. Frequency of recepient genome in selected plants was $16 \%-19 \%$ higher than the ordinary breeding.

- In BC2F1, three plants with the recipient alleles up to $94.03 \%$ and $93.18 \%$ were used to cross with recipeent variety for $\mathrm{BC} 3 \mathrm{~F} 1$ generation

- Plant P284-112-209 was the best BC3F1 individual with all the recipient alleles screened based on total of 63 markers. The four plants P307-305-21, P284-112195, P284-112-198, P284-112-213 were the second ranking with only one loci heterozygous. All those 5 plants were chosen as the breeding lines for result of Saltol-AS996 introgression

- Conventional breeding will be applied on the BC4F1, $\mathrm{BC} 3 \mathrm{~F} 2$ for selection of the new salt tolerance rice lines with all receipient genome. 


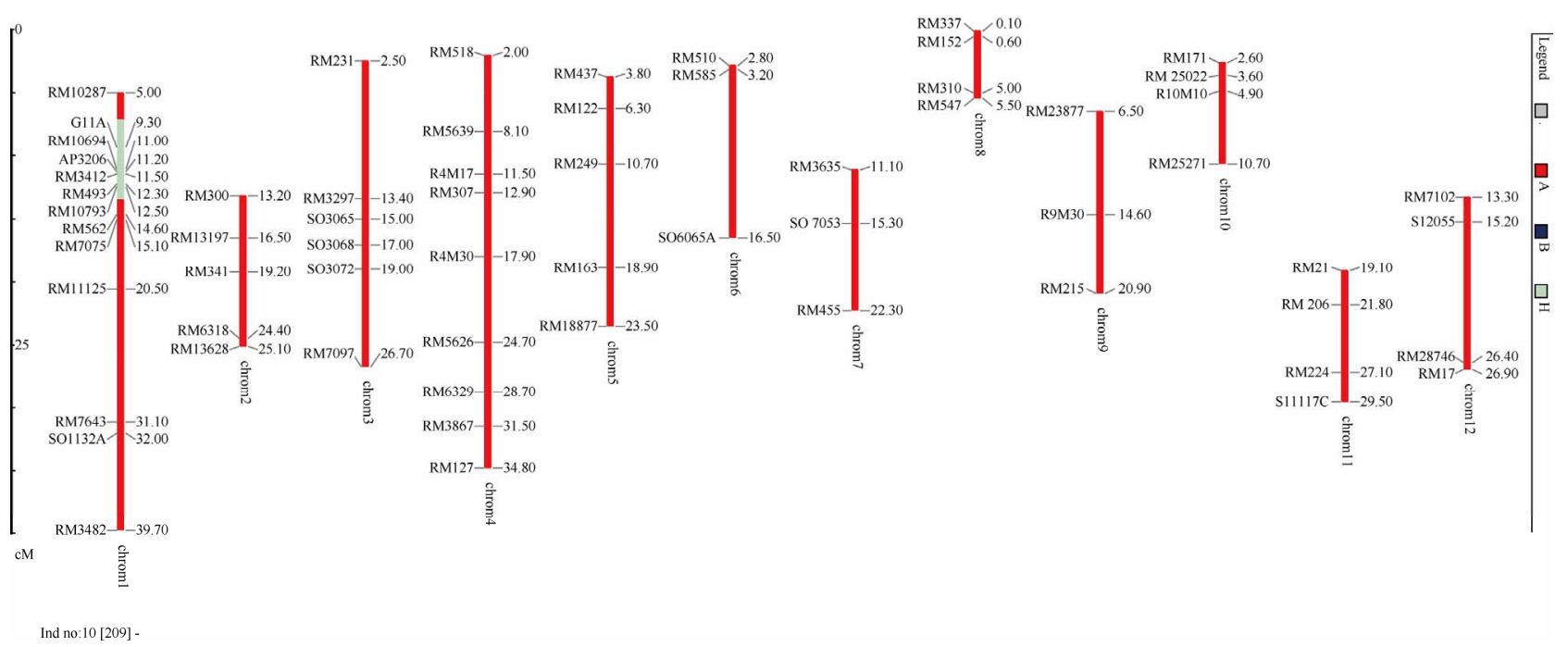

Figure 6. Graphical of the best plants BC3F1-P284-112-209 using GGT2.0.

\section{Acknowledgements}

Authors gratefully acknowledge Ms. Pham Thi Minh Hien, Mr. Nguyen Quang Dam for technical assistance and Dr. Pham Thi Mui for field works and crossing in this study. Thanks are due to Danish Ministry of Foreign Affair and DANIDA Fellowship for sponsoring the research projects "Improving Rice Tolerance of Submergence to cope with Climate Change in Coastal Areas of Vietnamese Deltas" (Project code: 09-P01-VIE). We also thank the staff of the International Rice Research Institute (IRRI)-Los Baños, Laguna, Philippines, for their colaboration in this research.

\section{REFERENCES}

[1] M. J. Thomson, A. M. Ismail, S. R. McCouch and M. J. Mackill, "Marker Assisted Breeding," In: A. Pareek, S. K. Sopory, H. J. Bohnert and Govindjee, Eds., Abiotic Stress Adaptation in Plants: Physiological, Molecular and Genomic Foundation, Springer, New York, 2010, pp. 451469.

[2] E. M. Septiningsih, A. M. Pamplona, D. L. Sanchez, C. N Neeraja, G. V. Vergara, S. Heuer, A. M. Ismail and D. J. Mackill, "Development of Submergence Tolerant Rice Cultivars: The Sub1 Locus and Beyond," Annals of Botany, Vol. 103, No. 2, 2009, pp. 151-160. doi:10.1093/aob/men206

[3] R. K. Singh, E. D. Redoña and L. Refuerzo, "Varietal Improvement for Abiotic Stress Tolerance in Crop Plants: Special Reference to Salinity in Rice," In: A. Pareek, S. K. Sopory, H. J. Bohnert and Govindjee, Eds., Abiotic Stress Adaptation in Plants: Physiological, Molecular and Genomic Foundation, Springer, New York, 2010, pp. $387-$ 415.

[4] B. C. Y. Collard and D. J. Mackill, "Marker-Assisted Selection: An Approach for Precision Plant Breeding in the 21st Century," Philosophical Transactions of the Royal Society, Vol. 363, No. 1491, 2008, pp. 557-572. doi:10.1098/rstb.2007.2170

[5] S.-H. Kim, P. R. Bhat, X. Cui, H. Walia, J. Xu, S. Wanamaker, et al., "Detection and Validation of Single Feature Polymorphisms Using RNA Expression Data from a Rice Genome Array," BMC Plant Biology, Vol. 9, 2009, p. 65. doi:10.1186/1471-2229-9-65

[6] R. Van Berloo "GGT 2.0: Versatile Software for Visualization and Analysis of Genetic Data," Journal of Heredity, Vol. 99, No. 2, 2008, pp. 232-236. doi:10.1093/jhered/esm109

[7] S. Yoshida, D. A. Forno, J. K. Cock and K. A. Gomez, "Laboratory Manual for Physiological Studies of Rice," International Rice Research Institute, Manila, 1976, p. 38.

[8] IRRI, "Standard Evaluation System for Rice," 4th Edition, International Rice Research Institute, Manila, 1996, p. 52. 